Cite as: E. S. Knock et al., Sci. Transl. Med. 10.1126/scitranslmed.abg4262 (2021).

\title{
CORONAVIRUS
}

\section{Key epidemiological drivers and impact of interventions in the 2020 SARS-CoV-2 epidemic in England}

\begin{abstract}
Edward S. Knock ${ }^{1,2 *}$, Lilith K. Whittles ${ }^{1,2,3 *}$, John A. Lees ${ }^{1 *}$, Pablo N. Perez-Guzman ${ }^{1 *}$, Robert Verity $^{1}$, Richard G. FitzJohn', Katy A.M. Gaythorpe ${ }^{1}$, Natsuko Imai', Wes Hinsley ${ }^{1}$, Lucy C. Okell ${ }^{1}$, Alicia Rosello ${ }^{4}$, Nikolas Kantas ${ }^{5}$, Caroline E. Walters ${ }^{1}$, Sangeeta Bhatia', Oliver J. Watson ${ }^{1}$, Charlie Whittaker ${ }^{1}$, Lorenzo Cattarino ${ }^{1}$, Adhiratha Boonyasiri $^{6}$, Bimandra A. Djaafara', Keith Fraser', Han Fu' ${ }^{1}$, Haowei Wang1, Xiaoyue Xi' ${ }^{5}$, Christl A. Donnelly $^{1,7,8}$, Elita Jauneikaite ${ }^{1}$, Daniel J. Laydon ${ }^{1}$, Peter J. White ${ }^{1,2,3}$, Azra C. Ghani', Neil M. Ferguson ${ }^{1,2}{ }^{\wedge}$, Anne Cori $^{1,2 \wedge}$, Marc Baguelinn',2,4

${ }^{1}$ MRC Centre for Global Infectious Disease Analysis, Abdul Latif Jameel Institute for Disease and Emergency Analytics (J-IDEA), School of Public Health, Imperial College London, London, W2 1PG, UK; UK. ${ }^{2}$ National Institute for Health Research (NIHR) Health Protection Research Unit (HPRU) in Modelling and Health Economics, UK. ${ }^{3}$ Modelling and Economics Unit, National Infection Service, Public Health England, London, NW9 5EQ, UK. ${ }^{4}$ Department of Infectious Disease Epidemiology, Faculty of Epidemiology and Population Health, London School of Hygiene and Tropical Medicine, London, WC1E 7HT, UK. ${ }^{5}$ Faculty of Natural Sciences, Department of Mathematics, Imperial College London, London, SW7 2BX, UK. 'Department of Infectious Disease, School of Public Health, Imperial College London, London, W2 1PG, UK; UK. ${ }^{7}$ Department of Statistics, University of Oxford, Oxford, OX1 3LB, UK. ${ }^{8}$ NIHR HPRU in Emerging and Zoonotic Infections, UK.

*Equal contribution, ^ Equal contribution

*Corresponding author. Email: m.baguelin@imperial.ac.uk, neil.ferguson@imperial.ac.uk
\end{abstract}

We fitted a model of SARS-CoV-2 transmission in care homes and the community to regional surveillance data for England. Compared with other approaches, our model provides a synthesis of multiple surveillance data streams into a single coherent modelling framework allowing transmission and severity to be disentangled from features of the surveillance system. Of the control measures implemented, only national lockdown brought the reproduction number $\left(R_{t}^{\text {eff }}\right)$ below 1 consistently; if introduced one week earlier it could have reduced deaths in the first wave from an estimated 48,600 to 25,600 (95\% credible interval [95\% Crl]: 15,900-38,400). The infection fatality ratio decreased from $1.00 \%(95 \% \mathrm{Crl}: 0.85 \%-1.21 \%)$ to $0.79 \%(95 \% \mathrm{Crl}: 0.63 \%-0.99 \%)$, suggesting improved clinical care. The infection fatality ratio was higher in the elderly residing in care homes $(23.3 \%, 95 \% \mathrm{Crl}: 14.7 \%-35.2 \%)$ than those residing in the community (7.9\%, 95\%Crl: 5.9\%-10.3\%). On 2nd December 2020 England was still far from herd immunity, with regional cumulative infection incidence between $7.6 \%$ (95\%Crl: 5.4\%-10.2\%) and 22.3\% (95\%Crl: $19.4 \%-$ $25.4 \%$ ) of the population. Therefore, any vaccination campaign will need to achieve high coverage and a high degree of protection in vaccinated individuals to allow non-pharmaceutical interventions to be lifted without a resurgence of transmission.

\section{INTRODUCTION}

England is among the countries worst-affected by the global pandemic of COVID-19, caused by the novel Betacoronavirus SARS-CoV-2. Over 66,000 deaths are reported to have occurred by $2^{\text {nd }}$ December 2020 in England, or 117 deaths per 100,000 people (1). The impact of the epidemic has varied across the country, with regional epidemics differing in their severity and timing. A key feature in all regions is the burden suffered by older adults living in care homes, where mortality has been high.

We used a mathematical model of SARS-CoV-2 transmission to reproduce the first two waves of the epidemic across England's seven NHS regions and assess the impact of interventions implemented by the UK government. We analyzed the epidemic from importation of SARS-CoV-2 into each region to the $2^{\text {nd }}$ December 2020, encompassing the first national lockdown from March - May 2020, the interventions implemented as COVID-19 deaths increased again in the autumn, and the second national lockdown in November.

We developed an age-structured stochastic transmission model of SARS-CoV-2, representing care homes, hospital clinical pathways, and the wider community. We used a Bayesian evidence-synthesis approach to estimate model parameters and to reconstruct regional epidemics using data from daily recorded deaths, PCR testing, hospital admissions, hospital bed occupancy, individual patient outcomes, contact surveys, and serological surveys. This approach, based on integrating multiple data streams into a single coherent modelling framework ensured robust epidemiological estimates where characteristics of transmission and severity of SARS-CoV-2 
could be disentangled from features of the surveillance system. We evaluated temporal changes in transmission as new control measures were implemented and then relaxed, and population immunity accrued. Inclusion of serological data (accounting for seroreversion) allowed us to robustly estimate region- and age-specific disease severity, to compare severity in care home residents to elderly individuals in the community, and to estimate the total epidemic size by calculating the proportion of individuals infected over time in each region. Last, we examined counterfactual epidemic scenarios, varying the date and duration of the first national lockdown and the effectiveness of restricting care home visits, to quantify the resulting impact on mortality.

Our analysis, which integrates multiple data sources and parametrically accounts for their biases, provides a balanced overview of transmission, hospitalisation, and mortality patterns of SARS-CoV-2 in the first and second waves (up to $2^{\text {nd }}$ December) in all regions of England. Our results provide crucial insights for controlling the epidemic in the future emphasizing the importance of acting fast to save lives.

\section{RESULTS}

\section{Epidemic trajectory}

We used estimates of clinical progression to parameterise a stochastic compartmental SEIR-like transmission model incorporating care-homes and hospitalisation pathways. Incorporating these estimates into our evidence-synthesis approach, we inferred the COVID-19 epidemic start date (assumed as the date when thirty asymptomatic infectious individuals was reached) in each NHS England region (Fig. 1A), then reconstructed epidemic trajectories for hospitalisations (Figure S1) and deaths in care homes and hospitals (Fig. 1BH). Noticeably, throughout England, deaths in care homes peaked on average 13 days later than hospital deaths (Fig. 1B$\mathrm{H})$.

We estimated the basic reproduction number $R_{0}$ (defined as the expected number of onward infections from an infectious individual in a fully susceptible population) to be 2.8 (95\% CrI: 2.5-3.3) nationally. Figure 1I shows how the effective reproduction number $R_{t}^{e f f}$ (the average number of secondary cases generated by an individual infected at time $t$ ) changed in each region over time in relation to government control measures and accrual of population immunity.

The first COVID-19 death in England occurred on $5^{\text {th }}$ March 2020 (2). Seven days later, in response to the growing epidemic, the government began to introduce control measures, initially requiring individuals with a dry persistent cough or fever to self-isolate (3). On $23^{\text {rd }}$ March this escalated to a full national lockdown (3). Irrespective of initial differences, the degree of transmission during lockdown was similar across all regions (Fig. 1I), consistent with mobility data showing movement during lockdown reduced to a consistent level nationally (4).

We estimated that the epidemic in London and the South East began approximately two weeks before the rest of the country (Fig. 1A), meaning that the lockdown occurred at a later stage of the epidemic in those areas. London experienced an estimated mortality of 96.3 (95\% CrI: 84.7-108.4) per 100,000 during the first wave, compared with the estimated national average of 86.4 (95\% CrI: 75.8-99.1), despite having a younger population and a smaller estimated care home population than other regions (294 vs 603 per 100,000 nationally) (5).

The first lockdown in England continued until $11^{\text {th }}$ May 2020, when people unable to work remotely were permitted to resume their jobs. Over the summer restrictions were successively eased, with non-essential shops, pubs, and restaurants opening, followed by the government's 'Eat Out to Help Out' restaurant subsidy scheme in August (6). This led to a steady increase in transmission, with $R_{t}^{\text {eff }}$ estimated to rise above 1 in all regions by mid-August (Fig. II).

In common with other European countries, a key feature of the first epidemic wave in England was the high death toll within care homes, which accounted for an estimated $28 \%$ of COVID-19 deaths as of $1^{\text {st }}$ August 2020. Although community transmission rates fell during lockdown, our model suggested transmission within care homes continued to rise, with infection risk peaking in care home residents between $31^{\text {st }}$ March in London and $20^{\text {th }}$ April in the East of England (Fig. 2A).

Increasing PCR test positivity marked the beginning of a second epidemic wave (Fig. 2B-H, fig. S6). The accompanying introduction of non-pharmaceutical interventions (NPIs) began with the "Rule of Six" (limiting social gatherings to 6 persons maximum) on $14^{\text {th }}$ September (7), followed by the localized tiered restrictions on $14^{\text {th }}$ October (8). These measures limited transmission in most regions, but our model suggests they were insufficient to reduce $R_{t}^{\text {eff }}$ below 1 (Fig. 1I). Consequently, on $31^{\text {st }}$ October, the government announced a second national lockdown, which lasted from $5^{\text {th }}$ November to $1^{\text {st }}$ December (9).

Restrictions during the second lockdown were less stringent than the first, with schools and some workplaces remaining open. This was reflected in $R_{t}^{\text {eff }}$ estimates of 0.88 (95\% CrI: $0.82-0.95)$ on $18^{\text {th }}$ November 2020 , the midpoint of the second lockdown, compared to $R_{t}^{e f f}=0.68$ (95\% CrI: 0.650.72) on $16^{\text {th }}$ April 2020, the midpoint of the first. We estimated that without the population immunity accrued during the first wave, contact rates during the second lockdown would have resulted in a reproduction number of $R_{t}=1.05$ (95\% CrI: 0.97-1.14). Hence, population immunity helped to reduce transmission below the critical threshold of $R_{t}^{e f f}=1$.

\section{Severity and hospitalisation}

COVID-19 manifests a broad spectrum of severity, from asymptomatic infection to life-threatening illness requiring 
intensive care. We estimated age-patterns of clinical progression in people admitted to hospital using individual-level data from 17,702 patients admitted between 18th March and 31st May 2020 (inclusive) in the COVID-19 Hospitalisation in England Surveillance System (CHESS, (10)). We derived estimates of the time spent in each stage of the hospital pathway (including general wards, ICU, and post-ICU stepdown care), as well as age-stratified probabilities of progression through that pathway (Fig. 3A-E and Figure S2). Accounting for differing lengths of stays given different outcomes, there were marked differences in average length of ICU stay for those who died in ICU, those who later died in stepdown care, and those who were discharged following stepdown care (Fig. 3F). Among patients over 65, we found the probability of admission to ICU decreased with increasing age. Thus, it is possible that older and more severely infected patients were directed to care on a general ward rather than admitted to ICU where the benefits of ventilation and the corresponding prognosis may not be better than with oxygen therapy in a general ward (11).

We used estimates of clinical progression to parameterise the transmission model, enabling us to infer temporal and regional differences in disease severity informed by local demography, observed daily hospital admissions, bed occupancy, and deaths. We measured severity of disease by the infection fatality ratio (IFR) and the infection hospitalisation ratio (IHR). The severity of disease increased with age in all regions with the steepest increase above 65 years (Fig. 4A-C), in line with observations worldwide (5). Regional estimates of age-aggregated disease severity depended on the population age distribution, which was similar in most regions of the country, except London, where the median age was 34.6 years (vs 39.5 years nationally). At the start of the first wave, London experienced an estimated IFR of $0.63 \%$ (95\% CrI: $0.52 \%-0.75 \%)$ compared to the estimated national average of 1.00\% (95\% CrI: $0.85 \%-1.21 \%$ ). The IHR in London was $1.94 \%$ (95\% CrI: $1.68 \%-2.25 \%$ ) compared to the national average of 2.55\%; (95\% CrI: 2.17\%-3.04\%) (Fig. 4D-E). Regional variation in the population age distribution did not fully account for differences in severity, with London still experiencing lower mortality when stratified by age (Fig. 4A-B). The oldest age group $(80+)$ in London had an estimated IFR of $4.7 \%$ (95\% CrI: $3.6 \%-6.2 \%$ ) compared to $10.7 \%$ (95\% CrI: $8.2 \%-$ $13.8 \%$ ) in the North East and Yorkshire.

We estimated temporal trends in the overall IFR for England by weighting regional estimates by incidence. At the start of the first wave, the estimated national IFR was $1.00 \%$ (95\% CrI: $0.85 \%-1.21 \%$ ) (Fig. $4 \mathrm{E}$ ), consistent with earlier reports from serology data alone (12). The national IFR initially appeared to increase as transmission widened from London to regions with older populations and greater disease severity. Over the first wave, the proportion of hospital admissions resulting in death decreased, likely due to improvements in clinical management and alleviation of capacity constraints (13), leading to an estimated national IFR of $0.79 \%$ (95\% CrI: $0.63 \%-0.99 \%)$ by the end of the first wave. The magnitude of the relative reduction in IFR over time varied between regions, from an estimated $29.8 \%$ (95\% CrI: $15.5 \%-42.4 \%)$ in the North West to $44.6 \%$ (95\% CrI: $28.4 \%-57.7 \%$ ) in East of England.

The inferred IFR was greater among care home residents (23.3\%, 95\% CrI: $14.7 \%-35.2 \%)$ than in the $80+$ in the community (7.9\%, 95\% CrI: 5.9\%-10.3\%, Fig. 4C). Many care home residents did not transfer into hospital, and instead died in the facilities where they lived, so conversely the inferred IHR was lower in care home residents (18.8\%, 95\% CrI: 4.9\%$34.6 \%)$ than in those aged $80+$ in the community $(30.7 \%, 95 \%$ CrI: $24.1 \%-38.9 \%)$. We present national estimates of severity at the end of the second wave, stratified by age and care home residency, in table $\mathrm{S} 9$.

\section{Epidemic size}

Data from repeated serological surveys of blood donors aged 17-65 informed our estimation of the total regional 2020 epidemic size (Fig. 5A-G), accounting for the imperfect sensitivity and specificity of serological tests alongside seroreversion (14). Seropositivity notably declined following the first wave in some regions (Fig. 5A-G). This reflected seroreversion (15), but also likely temporal trends in the composition of the surveyed population. Lockdown restrictions made attending blood donation centres difficult for all except key workers, who were more likely to have been infected (16), and may therefore be overrepresented in the sample of blood donors during the two lockdowns. The decline of seropositivity is modeled independently of population immunity, which was assumed not to wane over the study period.

The estimated cumulative proportion of the population ever infected with SARS-CoV-2 ranged from 7.6\% (95\% CrI: $5.4 \%-10.2 \%$ ) in the South West to $22.3 \%$ (95\% CrI: $19.4 \%-$ $25.4 \%$ ) in London (Fig. 5H). The increase in seropositivity lagged cumulative infections by two weeks, reflecting the time from infection to seroconversion.

The estimated proportion of care home residents ever infected with SARS-CoV-2 was $29.8 \%$ (95\% CrI: $17.6 \%-44.0 \%$ ), much higher than the $6.1 \%$ (95\% CrI: 5.0\%-7.2\%) estimated in $>80$-year-olds living in the community. This difference was consistent across most regions (Fig. $5 \mathrm{H}$ ) where regional differences in the estimated care home attack rates mirrored the patterns estimated in the general community, with regions with larger community epidemics also experiencing larger care home epidemics (Fig. 5I,J). In the East of England and the South East this pattern was decoupled, likely due to slight inconsistencies in how COVID-19 cases were reported between different datasets. 


\section{Impact of non-pharmaceutical interventions (NPIs)}

We explored counterfactual intervention scenarios and examined the potential impact on mortality of initiating the first national lockdown one week earlier or later; ending that lockdown two weeks earlier or later; and with $50 \%$ more or less restricted care home visits throughout the epidemic (Fig. 6 ). We found that the timing of the initial national lockdown was crucial in determining the eventual epidemic size in England. We estimated that locking down a week earlier could have reduced the first wave death toll (up to $1^{\text {st }}$ July 2020) from an estimated 48,600 to 25,600 (95\% CrI: $15,900-38,400$ ) whereas delaying lockdown by a week would have increased the number of deaths to 132,800 (95\% CrI: 91,900-180,700) (Fig. 6A). It should be noted that an earlier lockdown could also result in a larger second wave, which could then be avoided by moving autumn control measures earlier. The estimated impact of such an approach varied by region, with regions with less established epidemics at the time of the first lockdown more sensitive to the timing of the intervention (Figure S3A-B). Conversely, we estimated that locking down a week later may have increased deaths, with large variability by region, from $117 \%$ in London to $248 \%$ in the North East and Yorkshire but with very large uncertainty (Figure S3B). Initiating a lockdown to interrupt the exponential growth phase of an epidemic has a much greater impact on reducing total mortality than extending an existing lockdown. Due to this asymmetry, we estimated that relaxing the lockdown measures two weeks earlier could have increased deaths by 9,400 (95\% CrI: $-6,300-26,800)$ prior to $2^{\text {nd }}$ December. Conversely, relaxing measures later could have prevented 8,300 (95\% CrI: $1,100-14,100)$ deaths prior to $2^{\text {nd }}$ December (Fig. $6 \mathrm{~B})$.

We also explored counterfactual scenarios varying the extent of visit restrictions in care homes and estimated that reducing contact between the general population and care home residents by $50 \%$ would not have dramatically affected care home deaths. The fits to care home deaths have lower and slightly later peaks compared with the data (Fig. 6C). This may be due to the different transmission dynamics in care homes at this time of the epidemics with for example, some NHS patients being discharged to care homes without prior testing. We estimated deaths could have decreased by up to $30 \%$ or increased by up to $24 \%$ compared with the median fitted simulations (Fig. 6C).

\section{DISCUSSION}

We present a detailed overview of SARS-CoV-2 transmission, hospitalisation, mortality and intervention impact in the first two epidemic waves across all regions of England between March and December 2020. We successfully reproduced the transmission dynamics of the two epidemic waves, in terms of cases, PCR prevalence, seroprevalence, hospitalised cases (general wards and ICU), and deaths in hospitals and in care homes.

We estimated intense transmission was occurring in care homes even during the first national lockdown when $R_{t}^{\text {eff }}$ in the community was well below one in all regions (17-19). Combined with our counterfactual analysis of restricting visits, this suggests that reducing infections in care home residents is challenging. This highlights the difficulty of protecting care home residents from COVID-19: due to the necessarily close contact between staff and residents within a care home, once an outbreak has begun it is very difficult to reduce transmission, which overrides any impact of reducing the number of introductions $(20,21)$. The disproportionately high burden of COVID-19 mortality in care homes has been observed in many high-income countries $(22,23)$ with an estimated IFR between $20-40 \%$ amongst care home residents in France assuming individuals are 3.8 to 6.0 times more frail than the general population (24). Our results about transmission in care homes are mitigated by the difficulty of reproducing the dynamics of mortality in care homes in some regions where deaths were underestimated by the model. This might be due to the simple approach used to model care homes and the uncertainty in actual number of care homes deaths as shown in the discrepancy between confirmed deaths and deaths attributed to COVID-19.

We found that, consistent with existing literature both in the UK (25) and globally (24), disease severity increased markedly with age. Assessment of severity is complicated by the broad clinical spectrum of COVID-19 (26-28), the population age structure, and surveillance systems $(29,30)$. Here we provide updated severity estimates for England based on multiple contemporary data streams. We estimated considerable regional heterogeneity in infection severity, broadly consistent in the general population and in care homes for IFR and IHR. London experienced the lowest severity even after adjusting for its younger population. The estimated two-fold reduction over time in IFR cannot be explained solely by the introduction of dexamethasone which reduces mortality amongst ICU patients (31), but rather a combination of factors including improvements in clinical management, greater experience in treating patients in ICU, and alleviation of capacity constraints $(13,32)$.

Our analysis showed large regional variation in burden, especially in the first wave. This is likely due to the pattern of seeding and the timing of national lockdown relative to how advanced each region's epidemic was. Our counterfactual scenarios of initiating the first national lockdown one week earlier or later highlight the importance of early interventions to reduce overall mortality.

Studies of COVID-19 interventions have found the effectiveness of NPIs depend critically on the local context and when restrictions are implemented relative to how large the epidemic has grown. Across multiple countries, a 
combination of NPIs were necessary to limit SARS-CoV-2 transmission, with studies finding that curfews, lockdowns, and restricting social gatherings $(33,34)$ or school closures and limits on internal movement being the most effective in reducing transmission (35). Our finding that only national lockdown measures consistently reduced the $R_{t}^{\text {eff }}$ below one is in agreement with other UK-based studies $(36,37)$.

At the midpoint of the second lockdown, we estimated a higher $R_{t}^{\text {eff }}$ of 0.88 (95\% CrI: 0.82-0.95) compared to the midpoint of the first lockdown in April $2020\left(R_{t}^{e f f}=0.68,95 \% \mathrm{CrI}\right.$ : 0.65-0.72). Less stringent restrictions were in place in England at this time with schools remaining open. Another study also estimated a smaller impact on transmissibility during the second national lockdown in England compared to during the "circuit-breaker" implemented in Wales which coincided with school half-term holidays (37). Additionally, the emergence of the B.1.1.7 lineage which has an estimated 43$100 \%$ multiplicative transmission advantage, coinciding with the second lockdown may have contributed to the higher $R_{t}^{\text {eff }}$ at this time $(38,39)$.

Even assuming immunity did not wane during the first year of the epidemic, our estimates of cumulative incidence over time strongly support the hypothesis that the epidemic decline after the first national lockdown was due to NPIs, with immunity playing a minimal role (40). Population-level immunity was insufficient to prevent a second wave of infection in any region, illustrated by the increase in reported cases and deaths which prompted the second national lockdown (41). Considerable uncertainties remain about the duration of immunity. For example, the extent and duration of infection-induced immunity to SARS-CoV-2 and its relationship to seropositivity remains unclear. Related seasonal coronaviruses induce immunity that wanes in one or two years (42), though antibody titers following SARS-CoV-1 infection appear to decay more slowly (43). Although including such immunity would not affect our results, this renders long term predictions about the dynamics of SARS-CoV-2 challenging. Our estimated cumulative incidence over time strongly supports the hypothesis that the epidemic decline after the first national lockdown was due to NPIs, with immunity playing a minimal role (40). Population-level immunity was insufficient to prevent a second wave of infection in any region, illustrated by the increase in reported cases and deaths which prompted the second national lockdown (41).

With the authorisation of the first SARS-CoV-2 vaccines in December 2020, we entered a new phase in the control of the COVID-19 pandemic. However, our estimates of population immunity at 2nd December 2020 were low, with regional cumulative attack rates ranging from $7.9 \%$ to $22.5 \%$; therefore any vaccination campaign will need to achieve high coverage and a high degree of protection in vaccinated individuals to allow NPIs to be lifted without a resurgence of transmission. Although vaccinating the most vulnerable age and risk groups will considerably reduce the burden of COVID-19, a large proportion of younger age groups may also need to be vaccinated to reach the immunity threshold for control. Our high estimates of transmission in care homes imply the need for high vaccine uptake there.

Our work has a number of limitations due to simplifying assumptions in our analysis. First, due to the compartmental nature of the model, we did not explicitly model individual care homes, rather the regional care home sector as a whole. However, as care home workers may work across multiple facilities leading to within and between care home transmission, we do not expect the simplification to substantially affect our conclusions but it might have contributed to our difficult to reproduce the peak of transmission in care homes. Similarly, we did not model individual households or transmission within and between them. When assessing the impact of NPIs on transmission we therefore captured population averages, rather than the contribution of household and non-household contacts. Second, hospital-acquired infections may have contributed to overall transmission, especially around the peak of the epidemic, and to persistence of infection in England over the summer months $(44,45)$. Our model does not explicitly represent nosocomial transmission; therefore, such effects will be encompassed within our regional $R_{t}^{e f f}$ estimates. Third, each data stream was subject to competing biases, which we statistically accounted for as far as possible (supplementary materials section 1.1.2). A key strength of our evidence-synthesis approach is that we do not rely on any single data source, combining multiple perspectives to provide a robust overall picture of the epidemic. We model the epidemics in each NHS region in England independently without accounting for transmission between regions; however, the majority of movement will be within rather than between regions. Last, limitations of the data meant we could not consider spatial heterogeneity within regions.

Our analysis provides a detailed overview of transmission, hospitalisation, and mortality patterns of COVID-19 in the first and second waves of the epidemic in all regions of England, one of the European countries worst-affected by the pandemic. Integration of multiple data streams into a single coherent modelling framework enables us to disentangle transmission and severity from features of the surveillance system, provide robust estimates of the epidemiological characteristics of the COVID-19 epidemic in England, and paves the way toward better understanding the contribution of individual surveillance data streams to the assessment of policy questions. As nationwide vaccination programmes are rolled out, our results will help to inform how NPIs are applied in the future. 


\section{MATERIALS AND METHODS}

\section{Study design}

We developed a stochastic SEIR-like age-structured compartmental model of the transmission of SARS-CoV-2 in community and care homes in England 2020 with a detailed description of progression into hospital pathways following severe disease (see diagram in fig S4). Model parameters were fitted to epidemiological data, including hospital admissions and bed occupancy, ICU prevalence, deaths in the community/hospitals/care homes, pillar-2 PCR testing data, REACT community surveys, and blood donor serological data (see the graph of the functional relationships linking model outputs, data streams, and parameters in fig. S5). Parameters of the model were estimated and the posterior distributions of the inferred model parameters were used to compute the epidemiological outcomes relevant to the analysis and run the counterfactual scenarios.

\section{Statistical analysis}

We first analyzed a linelist of 17,702 patients requiring hospitalisation from the CHESS Surveillance System. Using a progression model fitted with MCMC we derived age-stratified estimates of hospital progression parameters (see details in supplementary materials and data files: support_progression.csv, support_severity.csv). These parameter estimates were then used as priors in the larger compartmental transmission model to infer population-level estimates of severity alongside the rest of the parameters from the model. Bayesian estimation of the parameters of our models was performed using particle MCMC for each of the seven NHS regions independently (more details are provided in the supplementary materials).

Effective reproduction numbers were estimated from the eigenvalues of the next generation matrix derived from the posterior distributions of the estimated model parameters. An additional analysis using the EpiEstim R package (46) was carried out to test the robustness of this approach (see supplementary materials).

\section{SUPPLEMENTARY MATERIALS}

stm.sciencemag.org/cgi/content/full/scitranslmed.abg4262/DC1

Materials and Methods

Supplementary Text

Figs. S1 to S16

Tables S1 to S9

Data files S1 to S4

\section{REFERENCES AND NOTES}

1. GOV.UK, Coronavirus (COVID-19) in the UK (2020)

2. E. Mahase, Covid-19: UK records first death, as world's cases exceed 100 000. BMJ 368, m943 (2020). doi:10.1136/bmi.m943 Medline

3. GOV.UK, Prime Minister's statement on coronavirus (COVID-19): 20 March 2020 GOV.UK

4. B. Jeffrey, C. E. Walters, K. E. C. Ainslie, O. Eales, C. Ciavarella, S. Bhatia, S. Hayes, M. Baguelin, A. Boonyasiri, N. F. Brazeau, G. Cuomo-Dannenburg, R. G. FitzJohn, K. Gaythorpe, W. Green, N. Imai, T. A. Mellan, S. Mishra, P. Nouvellet, H. J. T. Unwin, R. Verity, M. Vollmer, C. Whittaker, N. M. Ferguson, C. A. Donnelly, S. Riley,
Anonymised and aggregated crowd level mobility data from mobile phones suggests that initial compliance with COVID-19 social distancing interventions was high and geographically consistent across the UK. Wellcome Open Res. 5, 170 (2020). doi:10.12688/wellcomeopenres.15997.1 Medline

5. Care Quality Commission, [ARCHIVED CONTENT] UK Government Web Archive The National Archives.

6. HM Revenue \& Customs, Get a discount with the Eat Out to Help Out Scheme. www.gov.uk (2020).

7. GOV.UK, Rule of six comes into effect to tackle coronavirus - GOV.UK.

8. GOV.UK, Prime Minister announces new local COVID Alert Levels - GOV.UK.

9. GOV.UK, Prime Minister announces new national restrictions - GOV.UK.

10. NHS Digital, SGSS and CHESS data - NHS Digital.

11. NHS, Overview | COVID-19 rapid guideline: critical care in adults | Guidance | NICE.

12. N. F. Brazeau, R. Verity, S. Jenks, H. Fu, C. Whittaker, P. Winskill, I. Dorigatti, P. Walker, S. Riley, R. P. Schnekenberg, H. Hoeltgebaum, T. A. Mellan, S. Mishra, H. T. Juliette Unwin, O. J. Watson, Z. M. Cucunubá, M. Baguelin, L. Whittles, S. Bhatt, A. C. Ghani, N. M. Ferguson, L. C. Okell, Infection Fatality Ratio: Estimates from Seroprevalence, doi:10.25561/83545.

13. R. A. Armstrong, A. D. Kane, T. M. Cook, Outcomes from intensive care in patients with COVID-19: A systematic review and meta-analysis of observational studies. Anaesthesia 75, 1340-1349 (2020). doi:10.1111/anae.15201 Medline

14. Public Health England, Sero-surveillance of COVID-19 - GOV.UK

15. F. J. Ibarrondo, J. A. Fulcher, D. Goodman-Meza, J. Elliott, C. Hofmann, M. A. Hausner, K. G. Ferbas, N. H. Tobin, G. M. Aldrovandi, O. O. Yang, Rapid Decay of Anti-SARS-CoV-2 Antibodies in Persons with Mild Covid-19. N. Engl. J. Med. 383 1085-1087 (2020). doi:10.1056/NEJMc2025179 Medline

16. L. H. Nguyen, D. A. Drew, M. S. Graham, A. D. Joshi, C. G. Guo, W. Ma, R. S. Mehta, E. T. Warner, D. R. Sikavi, C. H. Lo, S. Kwon, M. Song, L. A. Mucci, M. J. Stampfer, W. C. Willett, A. H. Eliassen, J. E. Hart, J. E. Chavarro, J. W. Rich-Edwards, R. Davies, J. Capdevila, K. A. Lee, M. N. Lochlainn, T. Varsavsky, C. H. Sudre, M. J. Cardoso, J. Wolf, T. D. Spector, S. Ourselin, C. J. Steves, A. T. Chan, C. M. Albert, G. Andreotti, B. Bala, B. A. Balasubramanian, L. E. Beane-Freeman, J. S. Brownstein, F. J. Bruinsma, J. Coresh, R. Costa, A. N. Cowan, A. Deka, S. L. Deming-Halverson, M. Elena Martinez, M. E. Ernst, J. C. Figueiredo, P. Fortuna, P. W. Franks, L. B. Freeman, C. D. Gardner, I. M. Ghobrial, C. A. Haiman, J. E. Hall, J. H. Kang, B. Kirpach, K. C. Koenen, L. D. Kubzansky, J. V. Lacey Jr., L. Le Marchand, X. Lin, P. Lutsey, C. R. Marinac, M. E. Martinez, R. L. Milne, A. M. Murray, D. Nash, J. R. Palmer, A. V. Patel, E. Pierce, M. M. Robertson, L. Rosenberg, D. P. Sandler, S. H. Schurman, K. Sewalk, S. V. Sharma, C. J. Sidey-Gibbons, L. Slevin, J. W. Smoller, C. J. Steves, M. I. Tiirikainen, S. T. Weiss, L. R. Wilkens, F. Zhang; COronavirus Pandemic Epidemiology Consortium, Risk of COVID-19 among frontline health-care workers and the general community: A prospective cohort study. Lancet Public Health 5, e475-e483 (2020). doi:10.1016/S2468-2667(20)30164$X$ Medline

17. GOV.UK, COVID-19: number of outbreaks in care homes - management information

18. S. N. Ladhani, J. Y. Chow, R. Janarthanan, J. Fok, E. Crawley-Boevey, A. Vusirikala, E. Fernandez, M. S. Perez, S. Tang, K. Dun-Campbell, E. W. Evans, A. Bell, B. Patel, Z. Amin-Chowdhury, F. Aiano, K. Paranthaman, T. Ma, M. Saavedra-Campos, R. Myers, J. Ellis, A. Lackenby, R. Gopal, M. Patel, C. Brown, M. Chand, K. Brown, M. E. Ramsay, S. Hopkins, N. Shetty, M. Zambon, Investigation of SARS-CoV-2 outbreaks in six care homes in London, April 2020. EClinicalMedicine 26, 100533 (2020). doi:10.1016/i.eclinm.2020.100533 Medline

19. J. K. Burton, G. Bayne, C. Evans, F. Garbe, D. Gorman, N. Honhold, D. McCormick, R. Othieno, J. E. Stevenson, S. Swietlik, K. E. Templeton, M. Tranter, L. Willocks, B. Guthrie, Evolution and effects of COVID-19 outbreaks in care homes: A population analysis in 189 care homes in one geographical region of the UK. Lancet Heal. Longev. 1, e21-e31 (2020). doi:10.1016/S2666-7568(20)30012-X

20. L. J. Strausbaugh, S. R. Sukumar, C. L. Joseph, Infectious disease outbreaks in nursing homes: An unappreciated hazard for frail elderly persons. Clin. Infect. Dis. 36, 870-876 (2003). doi:10.1086/368197 Medline

21. T. Inns, D. Wilson, P. Manley, J. P. Harris, S. J. O'Brien, R. Vivancos, What proportion of care home outbreaks are caused by norovirus? An analysis of viral causes of gastroenteritis outbreaks in care homes, North East England, 20162018. BMC Infect. Dis. 20, 2 (2019). doi:10.1186/s12879-019-4726-4 Medline 
22. A. Declercq, M. De Stampa, L. Geffen, G. Heckman, J. Hirdes, H. Finne-Soveri, T. Lum, N. Millar, J. N. Morris, G. Onder, K. Szczerbińska, E. Topinkova, H. Van Hout, 'Why, in almost all countries, was residential care for older people so badly affected by COVID-19?'

23. S. Bagchi, J. Mak, Q. Li, E. Sheriff, E. Mungai, A. Anttila, M. M. Soe, J. R. Edwards, A. L. Benin, D. A. Pollock, E. Shulman, S. Ling, J. Moody-Williams, L. A. Fleisher, A. Srinivasan, J. M. Bell, Rates of COVID-19 Among Residents and Staff Members in Nursing Homes - United States, May 25-November 22, 2020. MMWR Morb. Mortal. Wkly. Rep. 70, 52-55 (2021). doi:10.15585/mmwr.mm7002e2 Medline

24. M. O'Driscoll, G. Ribeiro Dos Santos, L. Wang, D. A. T. Cummings, A. S. Azman, J. Paireau, A. Fontanet, S. Cauchemez, H. Salje, Age-specific mortality and immunity patterns of SARS-CoV-2. Nature 590, 140-145 (2021). doi:10.1038/s41586-0202918-0 Medline

25. P. N. Perez-Guzman, A. Daunt, S. Mukherjee, P. Crook, R. Forlano, M. D. Kont, A. Løchen, M. Vollmer, P. Middleton, R. Judge, C. Harlow, A. Soubieres, G. Cooke, P. J. White, T. B. Hallett, P. Aylin, N. Ferguson, K. Hauck, M. R. Thursz, S. Nayagam, Clinical characteristics and predictors of outcomes of hospitalized patients with COVID-19 in a multi-ethnic London NHS Trust: A retrospective cohort study. Clin. Infect. Dis. $\cdots, 1-11$ (2020). Medline

26. A. B. Docherty, E. M. Harrison, C. A. Green, H. E. Hardwick, R. Pius, L. Norman, K. A. Holden, J. M. Read, F. Dondelinger, G. Carson, L. Merson, J. Lee, D. Plotkin, L. Sigfrid, S. Halpin, C. Jackson, C. Gamble, P. W. Horby, J. S. Nguyen-Van-Tam, A. Ho, C. D. Russell, J. Dunning, P. J. M. Openshaw, J. K. Baillie, M. G. Semple; ISARIC4C investigators, Features of 20133 UK patients in hospital with covid-19 using the ISARIC WHO Clinical Characterisation Protocol: Prospective observational cohort study. BMJ 369, m1985 (2020). doi:10.1136/bmi.m1985 Medline

27. S. Tabata, K. Imai, S. Kawano, M. Ikeda, T. Kodama, K. Miyoshi, H. Obinata, S. Mimura, T. Kodera, M. Kitagaki, M. Sato, S. Suzuki, T. Ito, Y. Uwabe, K. Tamura, Clinical characteristics of COVID-19 in 104 people with SARS-CoV-2 infection on the Diamond Princess cruise ship: A retrospective analysis. Lancet Infect. Dis. 20, 1043-1050 (2020). doi:10.1016/S1473-3099(20)30482-5 Medline

28. P. Vetter, D. L. Vu, A. G. L'Huillier, M. Schibler, L. Kaiser, F. Jacquerioz, Clinical features of covid-19. BMJ 369, m1470 (2020). doi:10.1136/bmj.m1470 Medline

29. R. Verity, L. C. Okell, I. Dorigatti, P. Winskill, C. Whittaker, N. Imai, G. CuomoDannenburg, H. Thompson, P. G. T. Walker, H. Fu, A. Dighe, J. T. Griffin, M. Baguelin, S. Bhatia, A. Boonyasiri, A. Cori, Z. Cucunubá, R. FitzJohn, K. Gaythorpe, W. Green, A. Hamlet, W. Hinsley, D. Laydon, G. Nedjati-Gilani, S. Riley, S. van Elsland, E. Volz, H. Wang, Y. Wang, X. Xi, C. A. Donnelly, A. C. Ghani, N. M. Ferguson, Estimates of the severity of coronavirus disease 2019: A model-based analysis. Lancet Infect. Dis. 20, 669-677 (2020). doi:10.1016/S14733099(20)30243-7 Medline

30. A. T. Levin, W. P. Hanage, N. Owusu-Boaitey, K. B. Cochran, S. P. Walsh, G. Meyerowitz-Katz, Assessing the age specificity of infection fatality rates for COVID-19: Systematic review, meta-analysis, and public policy implications. Eur. J. Epidemiol. 35, 1123-1138 (2020). doi:10.1007/s10654-020-00698-1 Medline

31. The RECOVERY Collaborative Group, Dexamethasone in Hospitalized Patients with Covid-19- Preliminary Report. N. Engl. J. Med. 1-11 (2020).

32. L. I. Horwitz, S. A. Jones, R. J. Cerfolio, F. Francois, J. Greco, B. Rudy, C. M. Petrilli, Trends in COVID-19 Risk-Adjusted Mortality Rates. J. Hosp. Med. 23, 2020 (2020). Medline

33. N. Haug, L. Geyrhofer, A. Londei, E. Dervic, A. Desvars-Larrive, V. Loreto, B. Pinior, S. Thurner, P. Klimek, Ranking the effectiveness of worldwide COVID-19 government interventions. Nat. Hum. Behav. 4, 1303-1312 (2020). doi:10.1038/s41562-020-01009-0 Medline

34. S. Flaxman, S. Mishra, A. Gandy, H. J. T. Unwin, T. A. Mellan, H. Coupland, C. Whittaker, H. Zhu, T. Berah, J. W. Eaton, M. Monod, A. C. Ghani, C. A. Donnelly, S. Riley, M. A. C. Vollmer, N. M. Ferguson, L. C. Okell, S. Bhatt, L. Cattarino, L. V. Cooper, Z. Cucunubá, G. Cuomo-Dannenburg, A. Dighe, B. Djaafara, I. Dorigatti, S. L. van Elsland, R. G. FitzJohn, K. A. M. Gaythorpe, L. Geidelberg, N. C. Grassly, W. D. Green, T. Hallett, A. Hamlet, W. Hinsley, B. Jeffrey, E. Knock, D. J. Laydon, G. Nedjati-Gilani, P. Nouvellet, K. V. Parag, I. Siveroni, H. A. Thompson, R. Verity, E. Volz, C. E. Walters, H. Wang, Y. Wang, O. J. Watson, P. Winskill, X. Xi, P. G. T. Walker, A. C. Ghani, C. A. Donnelly, S. Riley, M. A. C. Vollmer, N. M. Ferguson, L. C. Okell, S. Bhatt; Imperial College COVID-19 Response Team, Estimating the effects of non-pharmaceutical interventions on COVID-19 in Europe. Nature 584, 257261 (2020). doi:10.1038/s41586-020-2405-7 Medline

35. Y. Liu, C. Morgenstern, J. Kelly, R. Lowe, M. Jit, C. J. Villabona-Arenas, H. Gibbs, C. A. B. Pearson, K. Prem, Q. J. Leclerc, S. R. Meakin, W. J. Edmunds, C. I. Jarvis, A. Gimma, S. Funk, M. Quaife, T. W. Russell, J. C. Emory, S. Abbott, J. Hellewell, D. C. Tully, R. M. G. J. Houben, K. O'Reilly, G. R. Gore-Langton, A. J. Kucharski, M. Auzenbergs, B. J. Quilty, T. Jombart, A. Rosello, O. Brady, K. E. Atkins, K. van Zandvoort, J. W. Rudge, A. Endo, K. Abbas, F. Y. Sun, S. R. Procter, S. Clifford, A. M. Foss, N. G. Davies, Y. W. D. Chan, C. Diamond, R. C. Barnard, R. M. Eggo, A. K. Deol, E. S. Nightingale, D. Simons, K. Sherratt, G. Medley, S. Hué, G. M. Knight, S. Flasche, N. I. Bosse, P. Klepac, M. Jit; CMMID COVID-19 Working Group, The impact of non-pharmaceutical interventions on SARS-CoV-2 transmission across 130 countries and territories. BMC Med. 19, 40 (2021). doi:10.1186/s12916-02001872-8 Medline

36. N. G. Davies, A. J. Kucharski, R. M. Eggo, A. Gimma, W. J. Edmunds, T. Jombart, K. O'Reilly, A. Endo, J. Hellewell, E. S. Nightingale, B. J. Quilty, C. I. Jarvis, T. W. Russell, P. Klepac, N. I. Bosse, S. Funk, S. Abbott, G. F. Medley, H. Gibbs, C. A. B. Pearson, S. Flasche, M. Jit, S. Clifford, K. Prem, C. Diamond, J. Emery, A. K. Deol, S. R. Procter, K. van Zandvoort, Y. F. Sun, J. D. Munday, A. Rosello, M. Auzenbergs, G. Knight, R. M. G. J. Houben, Y. Liu; Centre for the Mathematical Modelling of Infectious Diseases COVID-19 working group, Effects of non-pharmaceutical interventions on COVID-19 cases, deaths, and demand for hospital services in the UK: A modelling study. Lancet Public Health 5, e375-e385 (2020). doi:10.1016/S2468-2667(20)30133-X Medline

37. N. G. Davies, R. C. Barnard, C. I. Jarvis, T. W. Russell, M. G. Semple, M. Jit, W. J. Edmunds; Centre for Mathematical Modelling of Infectious Diseases COVID-19 Working Group; ISARIC4C investigators, Association of tiered restrictions and a second lockdown with COVID-19 deaths and hospital admissions in England: A modelling study. Lancet Infect. Dis. 21, 482-492 (2021). doi:10.1016/S14733099(20)30984-1 Medline

38. N. G. Davies, S. Abbott, R. C. Barnard, C. I. Jarvis, A. J. Kucharski, J. D. Munday, C. A. B. Pearson, T. W. Russell, D. C. Tully, A. D. Washburne, T. Wenseleers, A. Gimma, W. Waites, K. L. M. Wong, K. van Zandvoort, J. D. Silverman, K. Diaz-Ordaz, R. Keogh, R. M. Eggo, S. Funk, M. Jit, K. E. Atkins, W. J. Edmunds; CMMID COVID19 Working Group; COVID-19 Genomics UK (COG-UK) Consortium, Estimated transmissibility and impact of SARS-CoV-2 lineage B.1.1.7 in England. Science 372, eabg3055 (2021). doi:10.1126/science.abg3055 Medline

39. E. Volz, S. Mishra, M. Chand, J. C. Barrett, R. Johnson, L. Geidelberg, W. R. Hinsley, D. J. Laydon, G. Dabrera, Á. O'Toole, R. Amato, M. Ragonnet-Cronin, I. Harrison, B. Jackson, C. V. Ariani, O. Boyd, N. J. Loman, J. T. McCrone, S. Gonçalves, D. Jorgensen, R. Myers, V. Hill, D. K. Jackson, K. Gaythorpe, N. Groves, J. Sillitoe, D. P. Kwiatkowski, S. Flaxman, O. Ratmann, S. Bhatt, S. Hopkins, A. Gandy, A. Rambaut, N. M. Ferguson; COVID-19 Genomics UK (COG-UK) consortium, Assessing transmissibility of SARS-CoV-2 lineage B.1.1.7 in England. Nature 593, 266-269 (2021). doi:10.1038/s41586-021-03470-x Medline

40. L. C. Okell, R. Verity, O. J. Watson, S. Mishra, P. Walker, C. Whittaker, A. Katzourakis, C. A. Donnelly, S. Riley, A. C. Ghani, A. Gandy, S. Flaxman, N. M. Ferguson, S. Bhatt, Correspondence Have deaths from COVID-19 in Europe plateaued due to herd. Lancet 395, e110-e111 (2020). doi:10.1016/S01406736(20)31357-X Medline

41. Academy of Medical Science, Preparing for a challenging winter 2020/21, 79 (2020).

42. A. W. D. Edridge, J. Kaczorowska, A. C. R. Hoste, M. Bakker, M. Klein, K. Loens, M. F. Jebbink, A. Matser, C. M. Kinsella, P. Rueda, M. leven, H. Goossens, M. Prins, P. Sastre, M. Deijs, L. van der Hoek, Seasonal coronavirus protective immunity is short-lasting. Nat. Med. 26, 1691-1693 (2020). doi:10.1038/s41591-020-1083-1 Medline

43. S. M. Kissler, C. Tedijanto, E. Goldstein, Y. H. Grad, M. Lipsitch, Projecting the transmission dynamics of SARS-CoV-2 through the postpandemic period. Science 368, 860-868 (2020). doi:10.1126/science. abb5793 Medline

44. NHS England and NHS Improvement, 'Hospital Onset Covid-19: IPC evidence from recent survey and next steps'.

45. G. lacobucci, Covid-19: Doctors sound alarm over hospital transmissions. BMJ 369, m2013 (2020). doi:10.1136/bmi.m2013 Medline

46. A. Cori, EpiEstim: A Package to Estimate Time Varying Reproduction Numbers 
from Epidemic Curves. R Packag. version 2.2.4 (2021).

47. D. Buitrago-Garcia, D. Egli-Gany, M. J. Counotte, S. Hossmann, H. Imeri, A. M. Ipekci, G. Salanti, N. Low, Occurrence and transmission potential of asymptomatic and presymptomatic SARS-CoV-2 infections: A living systematic review and meta-analysis. PLOS Med. 17, e1003346 (2020). doi:10.1371/journal.pmed.1003346 Medline

48. S. Riley, C. E. Walters, H. Wang, O. Eales, K. E. C. Ainslie, C. Atchison, C. Fronterre, P. J. Diggle, D. Ashby, C. A. Donnelly, G. Cooke, W. Barclay, H. Ward, A. Darzi, P. Elliott, medRxiv, doi:10.1101/2020.12.15.20248244.

49. Office for National Statistics, Clinical commissioning group population estimates (National Statistics) - Office for National Statistics (2020).

50. GOV.UK, 'Care Homes Analysis Background' (2020).

51. Age UK, Later Life in the United Kingdom 2019.

52. J. Mossong, N. Hens, M. Jit, P. Beutels, K. Auranen, R. Mikolajczyk, M. Massari, S. Salmaso, G. S. Tomba, J. Wallinga, J. Heijne, M. Sadkowska-Todys, M. Rosinska, W. J. Edmunds, Social contacts and mixing patterns relevant to the spread of infectious diseases. PLOS Med. 5, e74 (2008). doi:10.1371/journal.pmed.0050074 Medline

53. S. Riley, O. Eales, H. Wang, C. Walters, R. FitzJohn, M. Whitaker, K. Ainslie, mrcide/reactidd: Journal resubmission (2021),, doi:10.5281/ZENOD0.4681283.

54. S. A. Lauer, K. H. Grantz, Q. Bi, F. K. Jones, Q. Zheng, H. R. Meredith, A. S. Azman, N. G. Reich, J. Lessler, The incubation period of coronavirus disease 2019 (CoVID19) from publicly reported confirmed cases: Estimation and application. Ann. Intern. Med. 172, 577-582 (2020). doi:10.7326/M20-0504 Medline

55. R. Fry, Personal communication (2021).

56. X. He, E. H. Y. Lau, P. Wu, X. Deng, J. Wang, X. Hao, Y. C. Lau, J. Y. Wong, Y. Guan, X. Tan, X. Mo, Y. Chen, B. Liao, W. Chen, F. Hu, Q. Zhang, M. Zhong, Y. Wu, L. Zhao, F. Zhang, B. J. Cowling, F. Li, G. M. Leung, Temporal dynamics in viral shedding and transmissibility of COVID-19. Nat. Med. 26, 672-675 (2020). doi:10.1038/s41591-020-0869-5 Medline

57. Q. Bi, Y. Wu, S. Mei, C. Ye, X. Zou, Z. Zhang, X. Liu, L. Wei, S. A. Truelove, T. Zhang, W. Gao, C. Cheng, X. Tang, X. Wu, Y. Wu, B. Sun, S. Huang, Y. Sun, J. Zhang, T. Ma, J. Lessler, T. Feng, Epidemiology and transmission of COVID-19 in 391 cases and 1286 of their close contacts in Shenzhen, China: A retrospective cohort study. Lancet Infect. Dis. 20, 911-919 (2020). doi:10.1016/S1473-3099(20)30287-5 Medline

58. A. Svensson, A note on generation times in epidemic models. Math. Biosci. 208, 300-311 (2007). doi:10.1016/i.mbs.2006.10.010 Medline

59. R. Harris, H. Whitaker, N. Andrews, F. Aiano, Z. Amin-Chowdhury, J. Flood, R. Borrow, E. Linley, S. Ahmad, L. Stapley, B. Hallis, G. Amirthalingam, K. Höschler, B. Parker, A. Horsley, T. Brooks, K. Brown, M. Ramsay, S. Ladhani, J. Infect., in press. 10.1101/2020.10.21.20216689

60. S. Omar, C. Bartz, S. Becker, S. Basenach, S. Pfeifer, C. Trapp, H. Hamm, H. C. Schlichting, M. Friederichs, U. Koch, C. Jestrabek, E. Hilger, M. Vogt, K. Jahn, S. Chen, T. Bärnighausen, P. Zanger; Palatina Public Health Study Group, Duration of SARS-CoV-2 RNA detection in COVID-19 patients in home isolation, RhinelandPalatinate, Germany, 2020 - an interval-censored survival analysis. Euro Surveill. 25, 1-8 (2020). doi:10.2807/1560-7917.ES.2020.25.30.2001292 Medline

61. B. Benny, G. Amandine, P. Kc, H. Sarah, M. Abby, C. Caitlin, S. Van, L.-S. James, 0. Affiliations, Quantifying antibody kinetics and RNA shedding during early-phase SARS-CoV-2 infection, doi:10.1101/2020.05.15.20103275.

62. S. Funk, Socialmixr: Social Mixing Matrices for Infectious Disease Modelling (2018).

63. S. Funk, socialmixr@ github.com.

64. GOV.UK, Prime Minister's statement on coronavirus (COVID-19): 12 March 2020 GOV.UK.

65. GOV.UK, Prime Minister's statement on coronavirus (COVID-19): 11 May 2020 GOV.UK.

66. GOV.UK, Prime Minister sets out timeline for retail to reopen in June - GOV.UK.

67. GOV.UK, Pubs, restaurants and hairdressers to reopen from 4 July - GOV.UK.

68. GOV.UK, Eat Out to Help Out launches today - with government paying half on restaurant bills - GOV.UK.

69. GOV.UK, Schools and colleges to reopen in full in September - GOV.UK.

70. P. Poletti, M. Tirani, D. Cereda, F. Trentini, G. Guzzetta, V. Marziano, S. Buoro, S. Riboli, L. Crottogini, R. Piccarreta, A. Piatti, G. Grasselli, A. Melegaro, M.
Gramegna, M. Ajelli, S. Merler, Age-specific SARS-CoV-2 infection fatality ratio and associated risk factors, Italy, February to April 2020. Euro Surveill. 25, 2001383 (2020). doi:10.2807/1560-7917.ES.2020.25.31.2001383 Medline

71. M. Bernabeu-Wittel, J. E. Ternero-Vega, P. Díaz-Jiménez, C. Conde-Guzmán, M. D. Nieto-Martín, L. Moreno-Gaviño, J. Delgado-Cuesta, M. Rincón-Gómez, L. Giménez-Miranda, M. D. Navarro-Amuedo, M. M. Muñoz-García, S. CalzónFernández, M. Ollero-Baturone, Death risk stratification in elderly patients with covid-19. A comparative cohort study in nursing homes outbreaks. Arch. Gerontol. Geriatr. 91, 104240 (2020). doi:10.1016/j.archger.2020.104240 Medline

72. NHS England and NHS Improvement, Statistics » COVID-19 Hospital Activity.

73. O. Diekmann, J. A. P. Heesterbeek, J. A. J. Metz, On the definition and the computation of the basic reproduction ratio $\mathrm{RO}$ in models for infectious diseases in heterogeneous populations. J. Math. Biol. 28, 365-382 (1990). doi:10.1007/BF00178324 Medline

74. A. Cori, N. M. Ferguson, C. Fraser, S. Cauchemez, A new framework and software to estimate time-varying reproduction numbers during epidemics. Am. J. Epidemiol. 178, 1505-1512 (2013). doi:10.1093/aje/kwt133 Medline

75. P. Nouvellet, S. Bhatia, A. Cori, K. E. C. Ainslie, M. Baguelin, S. Bhatt, A. Boonyasiri, N. F. Brazeau, L. Cattarino, L. V. Cooper, H. Coupland, Z. M. Cucunuba, G. CuomoDannenburg, A. Dighe, B. A. Djaafara, I. Dorigatti, O. D. Eales, S. L. van Elsland, F. F. Nascimento, R. G. FitzJohn, K. A. M. Gaythorpe, L. Geidelberg, W. D. Green, A. Hamlet, K. Hauck, W. Hinsley, N. Imai, B. Jeffrey, E. Knock, D. J. Laydon, J. A. Lees, T. Mangal, T. A. Mellan, G. Nedjati-Gilani, K. V. Parag, M. Pons-Salort, M. RagonnetCronin, S. Riley, H. J. T. Unwin, R. Verity, M. A. C. Vollmer, E. Volz, P. G. T. Walker, C. E. Walters, H. Wang, O. J. Watson, C. Whittaker, L. K. Whittles, X. Xi, N. M. Ferguson, C. A. Donnelly, Reduction in mobility and COVID-19 transmission. Nat. Commun. 12, 1090 (2021). doi:10.1038/s41467-021-21358-2 Medline

76. D. T. Gillespie, Approximate accelerated stochastic simulation of chemically reacting systems. J. Chem. Phys. 115, 1716-1733 (2001). doi:10.1063/1.1378322

77. Department of Health and Social Care, COVID-19 testing data: methodology note. www.gov.uk (2020).

78. I. M. C. Martin, C. A. Ison, D. M. Aanensen, K. A. Fenton, B. G. Spratt, Rapid sequence-based identification of gonococcal transmission clusters in a large metropolitan area. J. Infect. Dis. 189, 1497-1505 (2004). doi:10.1086/383047 Medline

79. P. Del Moral, A. Doucet, A. Jasra, Sequential Monte Carlo samplers. J. R. Stat. Soc. Series B Stat. Methodol. 68, 411-436 (2006). doi:10.1111/i.1467$9868.2006 .00553 . x$

80. C. Andrieu, A. Doucet, R. Holenstein, Particle Markov chain Monte Carlo methods. J. R. Stat. Soc. Series B Stat. Methodol. 72, 269-342 (2010). doi:10.1111/j.1467$9868.2009 .00736 . x$

81. N. J. Gordon, D. J. Salmond, A. F. M. Smith, Novel approach to nonlinear/nongaussian Bayesian state estimation. IEE Proceedings, Part F Radar Signal Process. 140, 107-113 (1993)

82. M. Baguelin, E. Knock, L. K. Whittles, R. FitzJohn, J. Lees, sircovid (2020).

83. E. S. Knock, L. K. Whittles, P. N. Perez-Guzman, S. Bhatia, F. Guntoro, O. J. Watson, C. Whittaker, N. M. Ferguson, A. Cori, M. Baguelin, R. G. FitzJohn, J. A. Lees, Reproducible parallel inference and simulation of stochastic state space models using odin, dust, and mcstate. Wellcome Open Res. 5, 288 (2020). doi:10.12688/wellcomeopenres.16466.1

84. R. Verity, R. FitzJohn, mrc-ide/markovid at version1.5.

85. C. I. Jarvis, K. Van Zandvoort, A. Gimma, K. Prem, P. Klepac, G. J. Rubin, W. J. Edmunds, J. C. Emery, R. M. G. J. Houben, N. Davies, E. S. Nightingale, S. Flasche, T. Jombart, J. Hellewell, S. Abbott, J. D. Munday, N. I. Bosse, S. Funk, F. Sun, A. Endo, A. Rosello, S. R. Procter, A. J. Kucharski, T. W. Russell, G. Knight, H. Gibbs, Q. Leclerc, B. J. Quilty, C. Diamond, Y. Liu, M. Jit, S. Clifford, C. A. B. Pearson, R. M. Eggo, A. K. Deol, P. Klepac, G. J. Rubin, W. J. Edmunds; CMMID COVID-19 working group, Quantifying the impact of physical distance measures on the transmission of COVID-19 in the UK. BMC Med. 18, 124 (2020). doi:10.1186/s12916-020-01597-8 Medline

Acknowledgments: We thank all the colleagues at Public Health England (PHE) and frontline health professionals who have driven and continue to drive the daily response to the epidemic, but also for providing the necessary data to inform this study. This work would not have been possible without their dedication and expertise. The use of pillar-2 PCR testing data was made possible thanks to PHE 
colleagues and we extend our thanks to Dr Nick Gent and Dr André Charlett for facilitation and their insights into these data. The use of serological data was made possible by colleagues at PHE Porton Down, Colindale, and the NHS Blood Transfusion Service. We are particularly grateful to Dr Gayatri Amirthalingam and Prof Nick Andrews for helpful discussions around these data. We thank all the REal-time Assessment of Community Transmission (REACT) Study investigators for kindly sharing their PCR prevalence data. We also thank the entire Imperial College London Covid-19 Response Team for their support and feedback throughout. The views expressed are those of the authors and not necessarily those of the United Kingdom (UK) Department of Health and Social Care, the National Health Service, the National Institute for Health Research (NIHR), Public Health England (PHE), UK MRC, UKRI or European Union.

Funding: This work was supported by the NIHR HPRU in Modelling and Health Economics, a partnership between PHE, Imperial College London and LSHTM (NMF, grant code NIHR200908) and the NIHR HPRU in Emerging and Zoonotic Infections, a partnership between PHE, University of Liverpool, University of Oxford and Liverpool School of Tropical Medicine (CAD, grant code NIHR200907). We acknowledge funding from the MRC Centre for Global Infectious Disease Analysis (NMF, reference MR/R015600/1), jointly funded by the UK Medical Research Council (MRC) and the UK Foreign, Commonwealth \& Development Office (FCDO), under the MRC/FCDO Concordat agreement and is also part of the EDCTP2 program supported by the European Union. Author contributions: ESK, LKW, JAL, AR, NK, PJW, ACG, NMF, and MB contributed to the original ideas and concepts; ESK, LKW, JAL, PJW, ACG, NMF, and MB formulated the overarching research goals and aims; ESK, LKW, JAL, PNPG, RV, RGF, LCO, and MB performed the formal analysis through application of statistical, mathematical and computational; ESK, LKW, JAL, RGF, KAMG, and $M B$ conducted the research and investigation process, specifically performing the experiments, or data/evidence collection; ESK, LKW, JAL, PNPG, RGF, NK, $C A D, N M F$, and MB developed or designed the methodology; ESK, LKW, JAL, PNPG, RGF, AR, CAD, NMF, and MB created the models; ESK, LKW, JAL, PNPG, RGF, WH, SB, OJW, CW, and MB implemented the computer code and supporting algorithms, including testing; ESK, LKW, JAL, KAMG, NI, AR, NK, CEW, OJW, HF, CAD, EJ, DJL, PJW, NMF, AC, and MB contributed to the manuscript creation including pre- or post-publication stages; LKW, JAL, RGF, $A R, N K, H W, X X, P J W, A C G, N M F$, and MB validated and verified the results and ensured of their reproducibility; LKW, JAL, PNPG, and RV prepared the visual material; LKW, PNPG, RV, AC, and MB wrote the original draft; LKW, PNPG, RV, RGF, KAMG, NI, WH, LCO, CW, LC, AB, BAD, KF, and HF collected and curated the data; JAL provided some computing resources; RGF, NI, and $\mathrm{MB}$ were in charge of project managing and coordination responsibility for the research activity planning and execution; PJW, NMF, AC, and MB acquired the funding for the project leading to this publication; ACG, NMF, AC, and MB carried oversight and leadership responsibility for the research activity planning and execution, including mentorship external to the core team. Competing interests: PJW and $\mathrm{AC}$ have received payment from Pfizer for teaching of mathematical modelling of infectious disease transmission and vaccination. The other authors declare that they have no competing interests. Data and materials availability: All code and de-identified regionally aggregated data (see supplementary materials for full details) required to reproduce this analysis are available at

https://zenodo.org/record/4384864. Four de-identified regionally aggregated data files are available in the supplementary materials. This work is licensed under a Creative Commons Attribution 4.0 International (CC BY 4.0) license, which permits unrestricted use, distribution, and reproduction in any medium, provided the original work is properly cited. To view a copy of this license, visit https://creativecommons.org/licenses/by/4.0/. This license does not apply to figures/photos/artwork or other content included in the article that is credited to a third party; obtain authorization from the rights holder before using this material.

Submitted 14 April 2021

Accepted 16 June 2021

Published First Release 22 June 2021

10.1126/scitranslmed.abg4262 


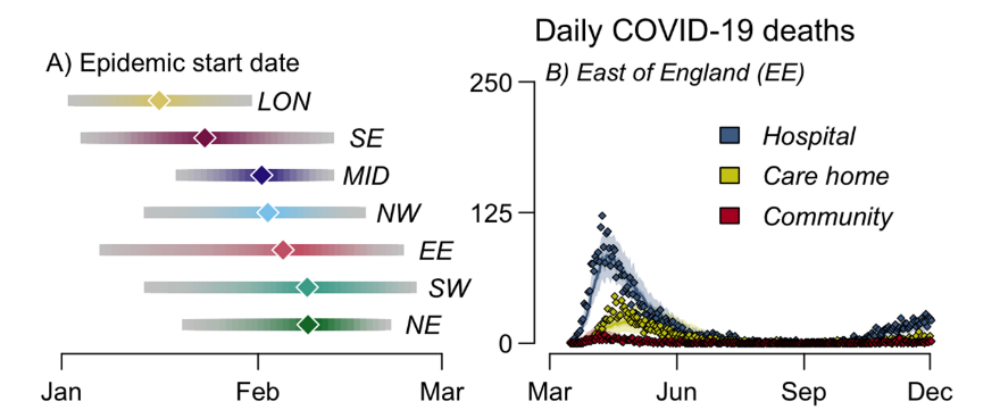

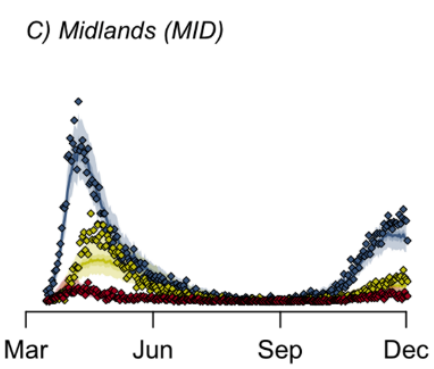

G) South East (SE)

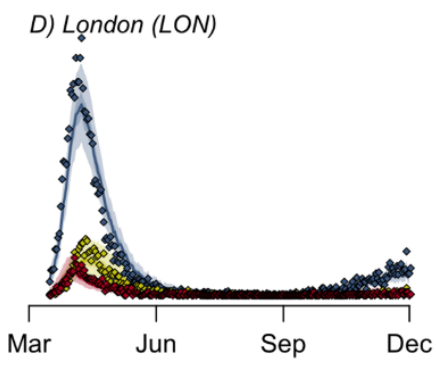

H) South West (SW)

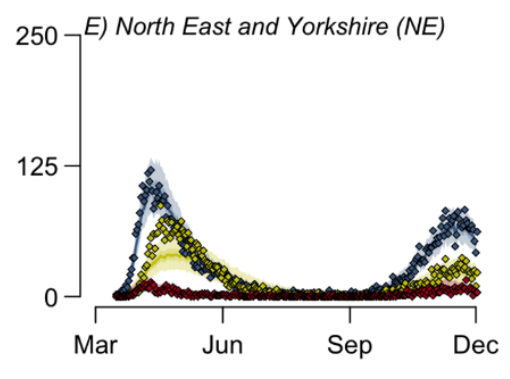

F) North West (NW)
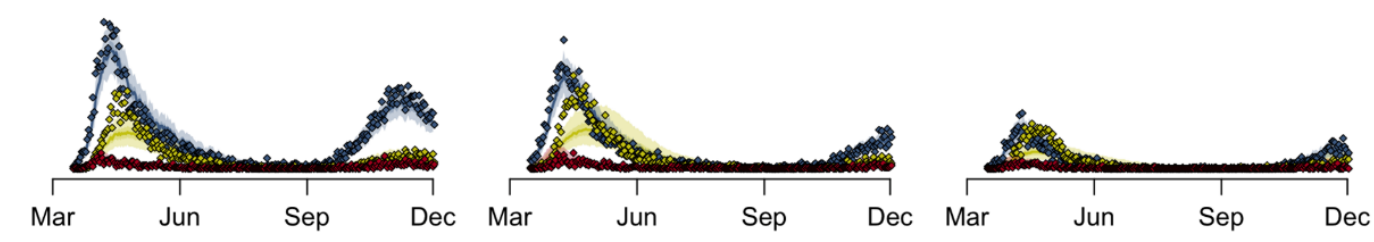

I) Transmission over time: $R_{t}^{\text {eff }}$

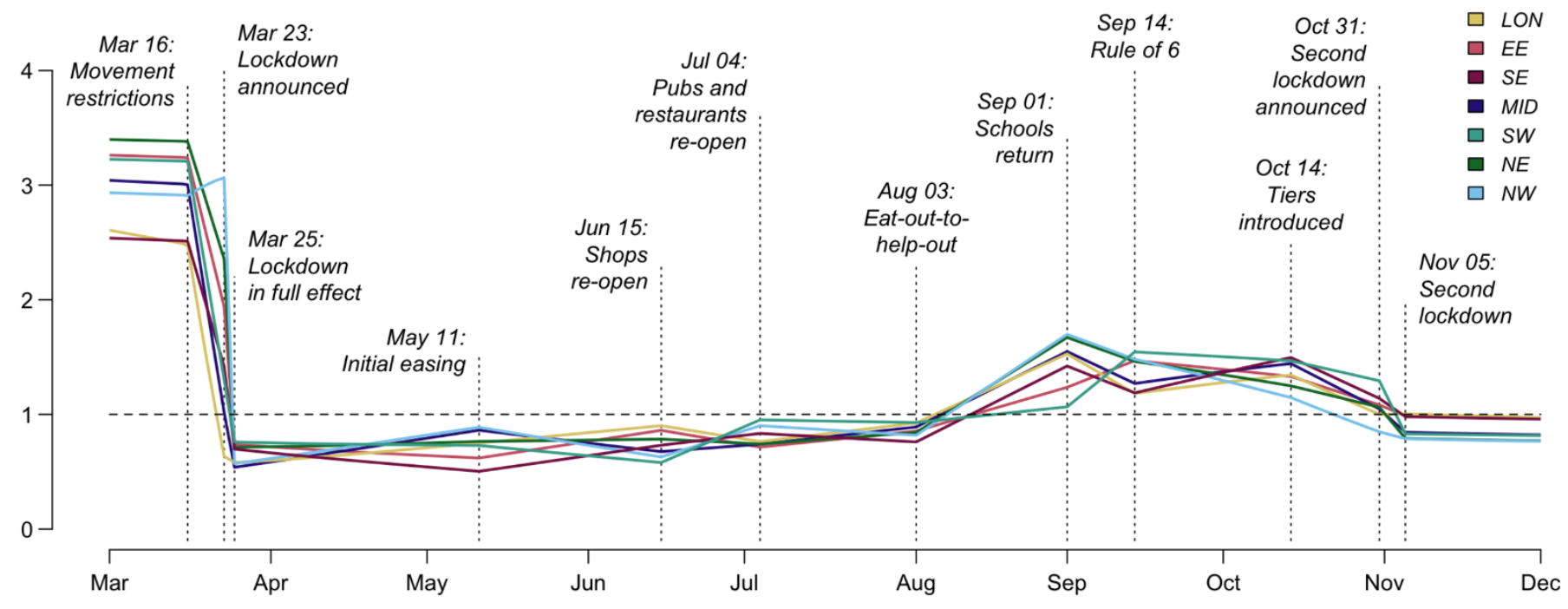

Fig. 1. Trajectory of the England COVID-19 epidemic. (A) Inferred epidemic start date in each NHS England region. (B-H) Model fit to reported daily deaths from COVID-19 in care homes, hospitals and in the community (that is, neither in a hospital or a care home) for each NHS England region. Points show the daily data (see supplementary materials section $\mathbf{1 . 1 . 2}$ for details of data sources) $(1)(<i>1</ i>)(<i>1</ i>)$. Solid lines the median posterior and shaded area shows the $95 \% \mathrm{Crl}$. (I) Mean estimated effective reproduction number within the general community (excluding care homes) in each region from March to December 2020. Vertical lines and labels represent dates of key policy changes, defining the breaking points of the underlying piecewise linear transmission rate. Dashed horizontal line depicts a reproduction number $\left(R_{0}\right)$ of 1 . 
A) England daily COVID-19 infections
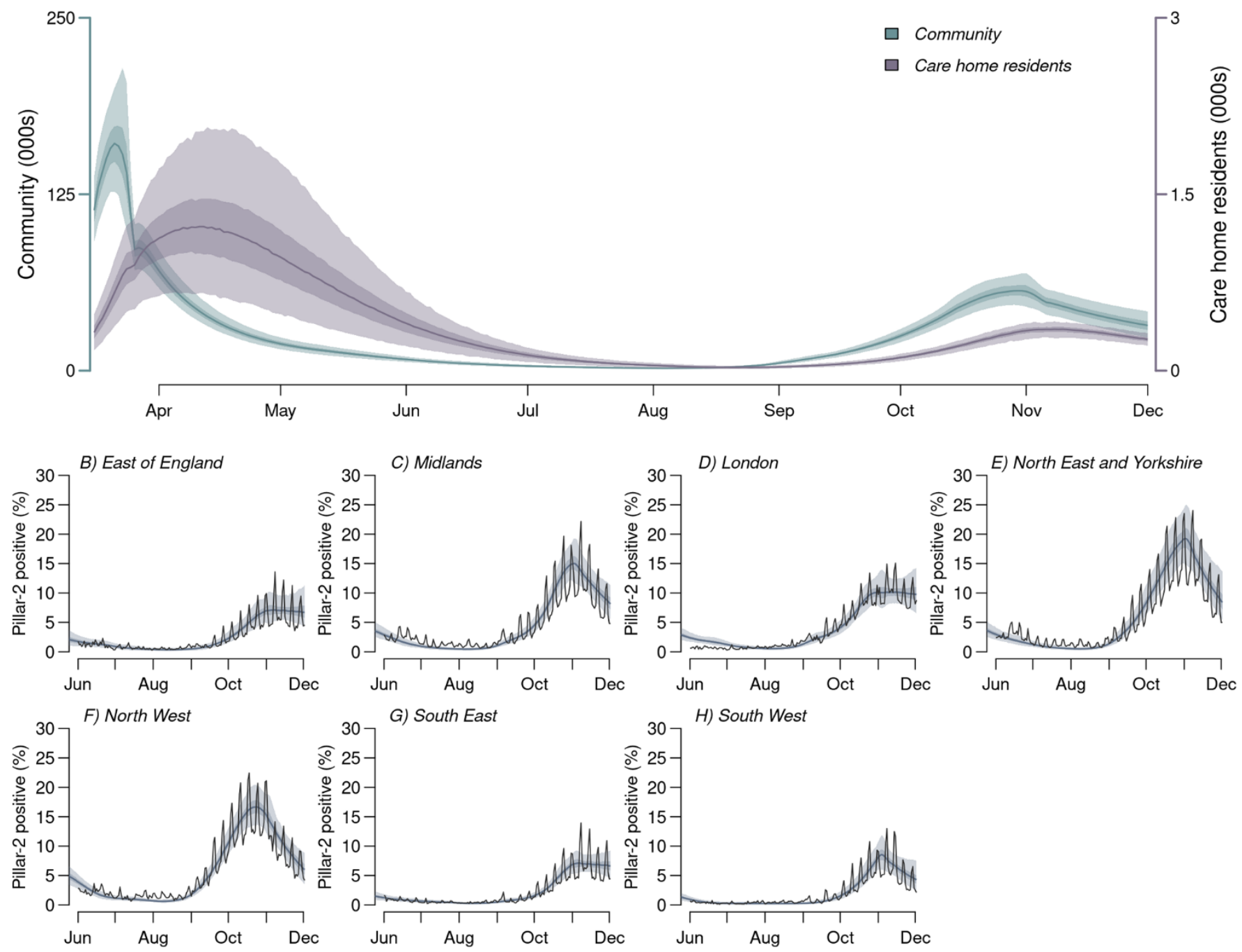

Fig. 2. Infection incidence and case positivity over time. (A) Inferred daily SARS-CoV-2 infections in England care home residents (excluding care home workers; right axis) and the wider community (left axis). (B-H) Comparison of modeled (shaded bands) and observed (solid line) proportion of PCR tests that were positive under pillar-2 testing (community swab testing for symptomatic individuals) in $>25$-year-olds. Shaded bands depict $95 \% \mathrm{Crl}, 50 \% \mathrm{Crl}$, and median model outputs. 


\section{Patient progression in hospital}

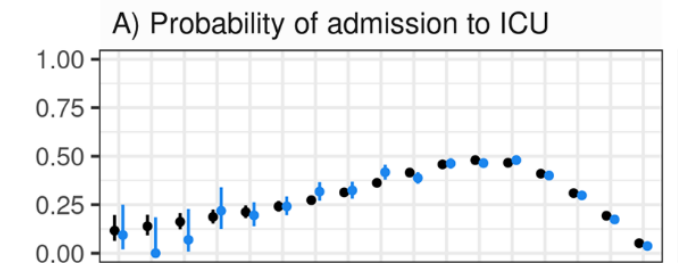

C) Probability of death in ICU

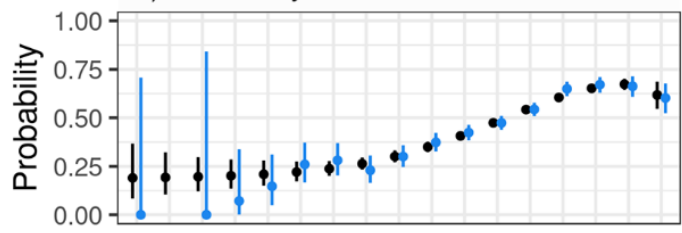

E) Probability of death by all pathways

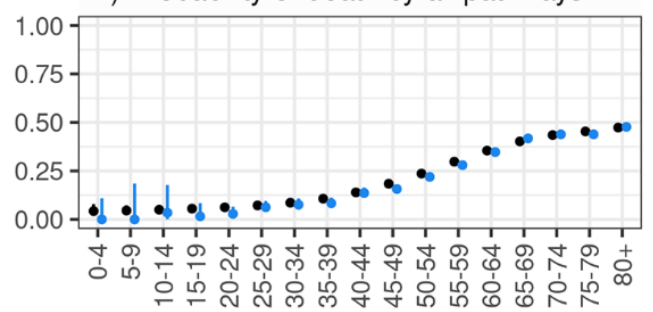

Age in years
B) Probability of death in general ward

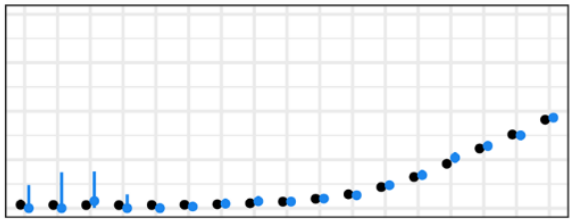

D) Probability of death in stepdown care

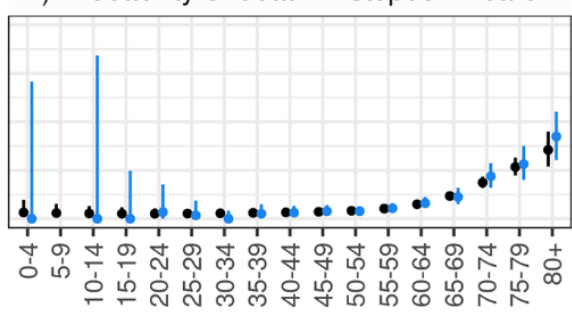

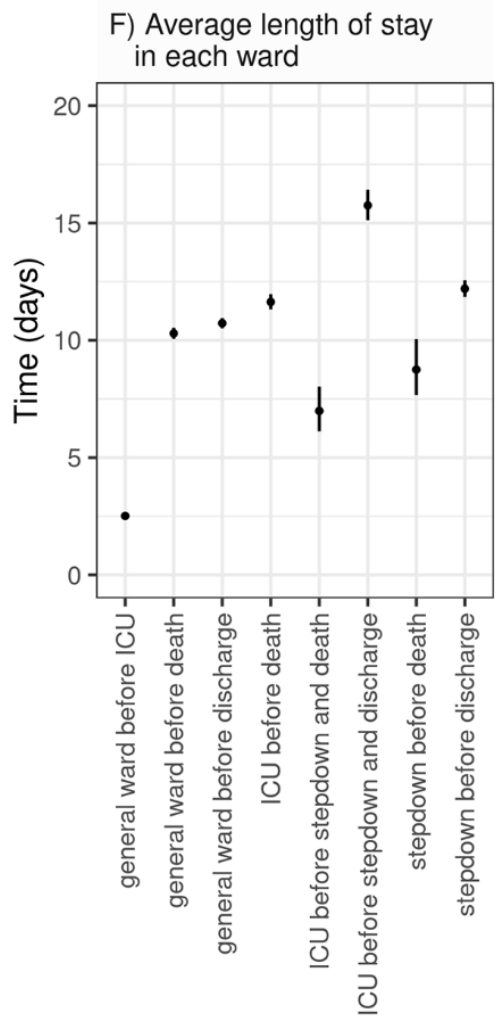

\$ Fitted spline intervals

Data binomial intervals

Fig. 3. Age-dependent probabilities of progression through hospital pathways. (A) Probability of admission to ICU. (B) Probability of death in a general hospital ward. (C) Probability of death in an ICU. (D) Probability of death in hospital during stepdown care. (E) Probability of death through all hospital pathways (obtained by combining B-D using the branching structure shown in fig. S4). Black circles and vertical segments show posterior mean and $95 \% \mathrm{Crl}$ of splines fitted to data, blue circles and vertical segments show raw data mean values and 95\% confidence intervals (exact binomial) for each 5-year age group. (F) Average estimated length of stay in each ward (posterior mean and 95\% Crl). 
A) Regional IHR by age

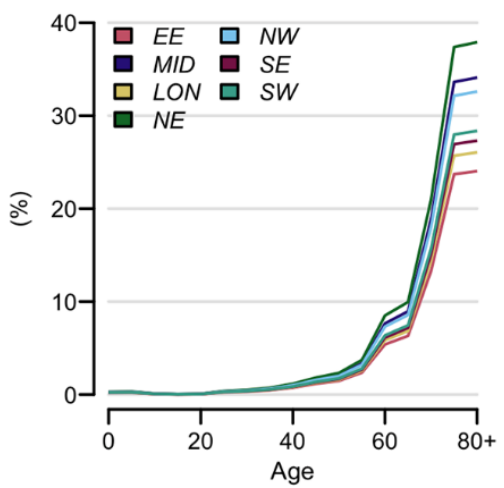

D) Regional IHR

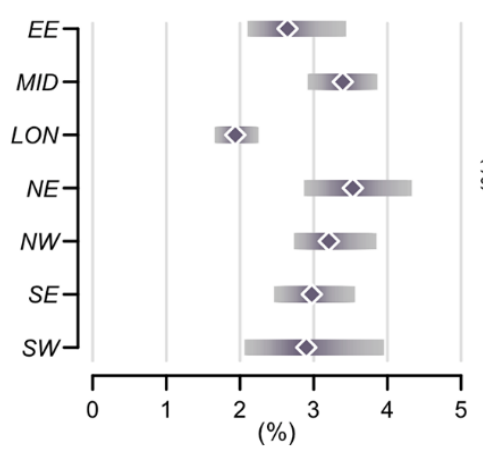

B) Regional IFR by age

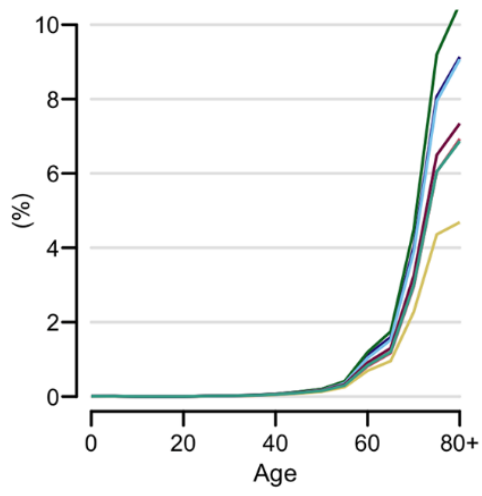

E) England age-aggregated IFR
C) England severity by age and in care home residents
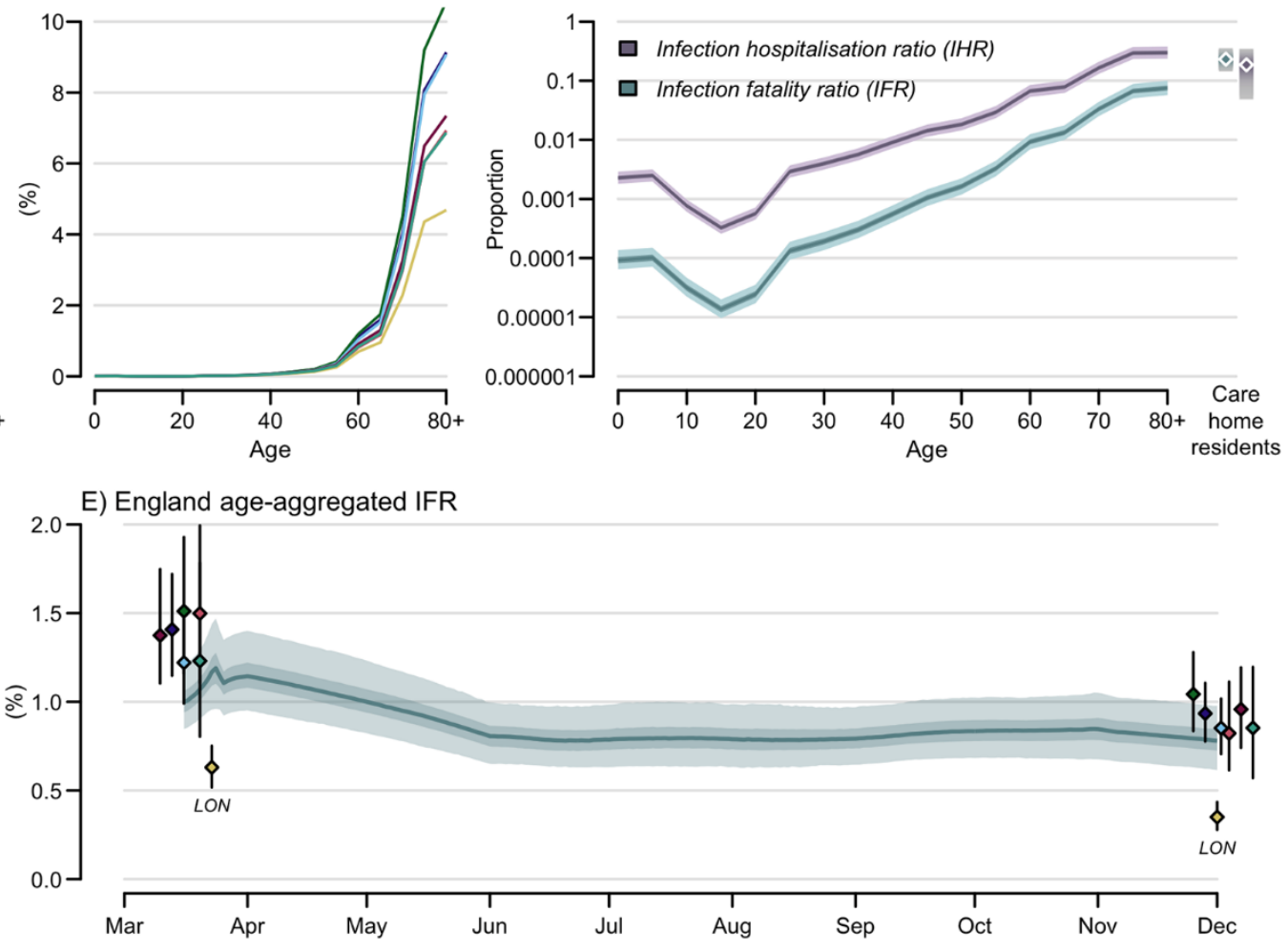

Fig. 4. Estimated relative severity of disease by age group and region. ( $A$ and $B$ ) Variation in $(A)$ the median inferred Infection fatality ratio (IFR) and (B) infection hospitalisation ratio (IHR) by age group in each region. Ages 80+ were modeled as a single risk group; care home residents were not included. (C) Estimated England IFR and IHR by age group and in care home residents (estimate excludes care home workers). National severity estimates are produced by aggregating regional estimates based on infection incidence. (D) Regional estimated IHR, aggregated over age and risk group by infection incidence. Plots in (A-D) use parameter estimates and incidence weightings calculated as of $1^{\text {st }}$ December 2020. (E) Estimated England IFR over time, colored dots show regional estimates of IFR at the start of the epidemic and on $1^{\text {st }}$ December 2020 (clusters each correspond to one time-point, LON: London). In (C-E) shaded bands depict 95\% Crl and interquartile ranges, points depict medians. 

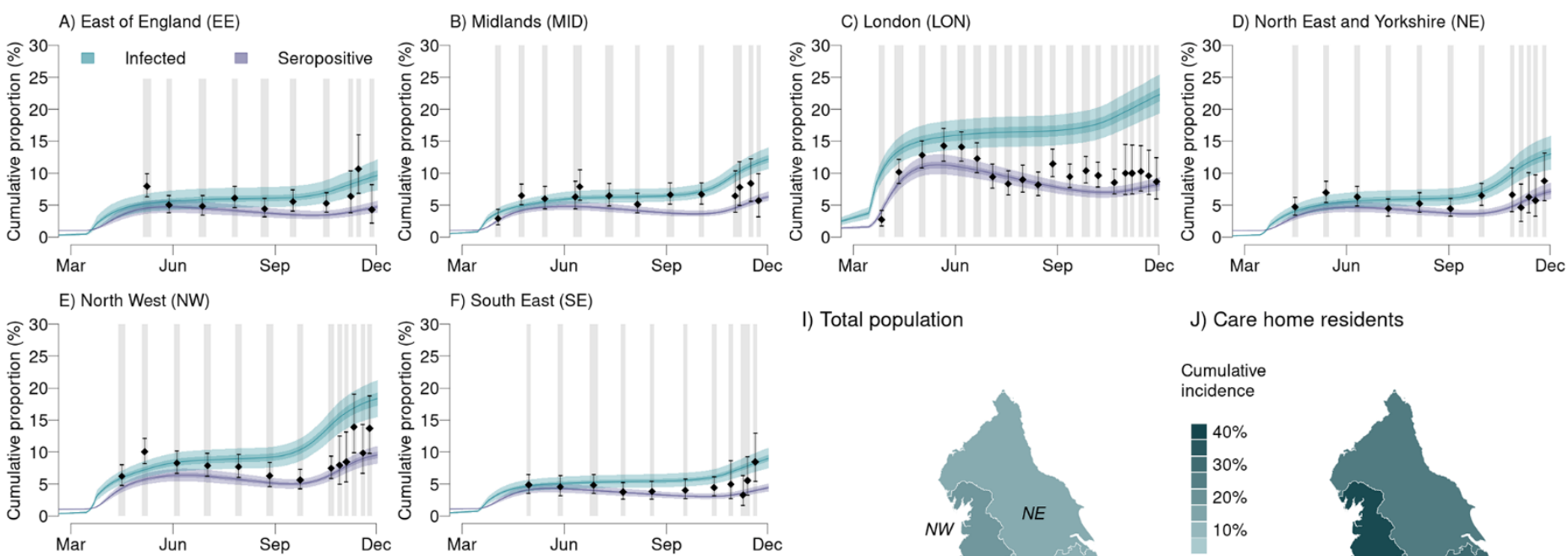

I) Total population

J) Care home residents
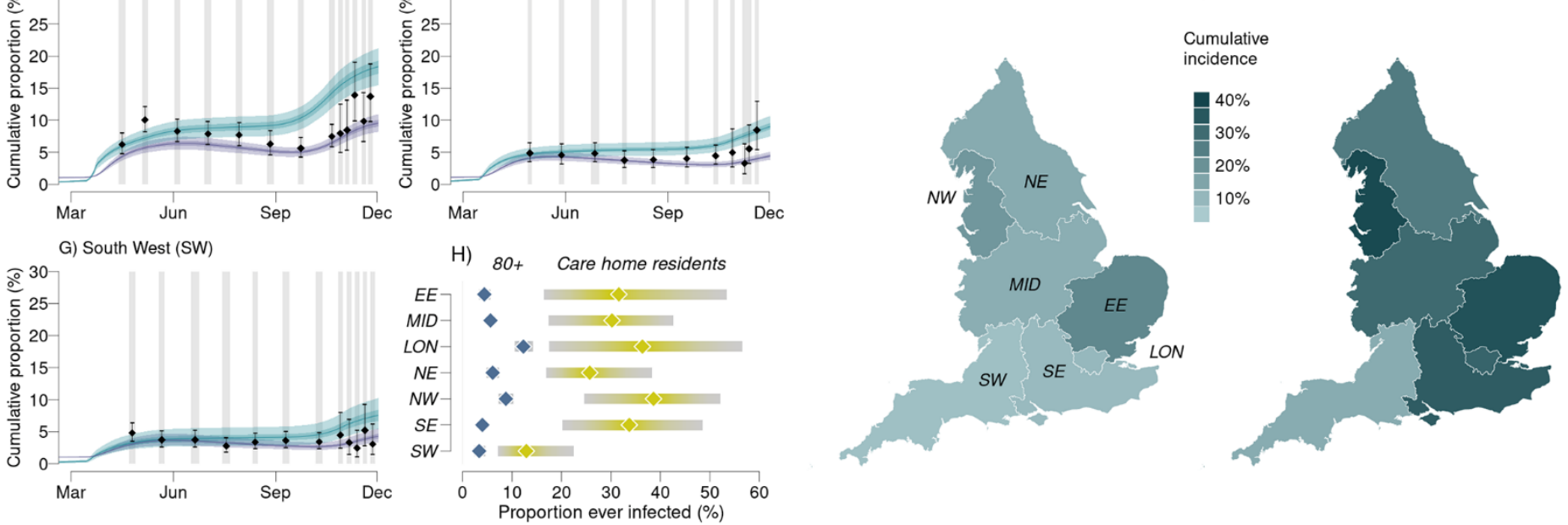

Fig. 5. Cumulative COVID-19 incidence and seropositivity by region. (A-G) Comparison of the estimated proportion of the population testing seropositive in 2020 with observations from serological surveys (see supplementary materials section 1.1.4 (14)). Vertical grey shaded bands show serological survey timings, black points the observed seroprevalence (bars: 95\% exact confidence intervals), blue and purple lines show the proportion of the population infected and seropositive respectively as inferred from our model (shaded bands the 95\% Crl, 50\% Crl and median). (H) Comparison by region of the estimated cumulative attack rate (median and $95 \% \mathrm{Crl}$ ) in care home residents (yellow, excludes care home workers) vs in the $80+$ age group in the community (blue). The estimated mean final epidemic size in each England NHS region I) in total and $\mathrm{J}$ ) in care home residents (excludes care home workers). 
A) Lockdown 1 week earlier

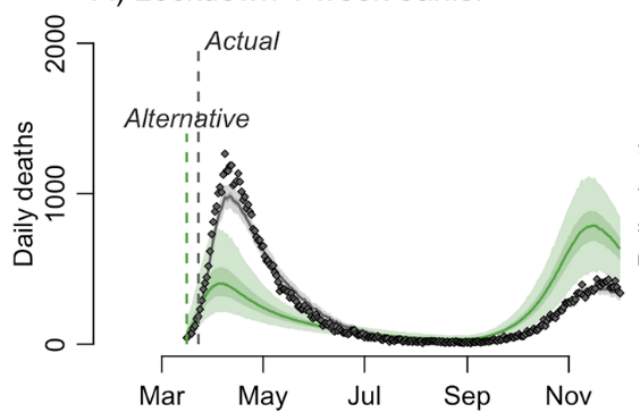

Lockdown 1 week later

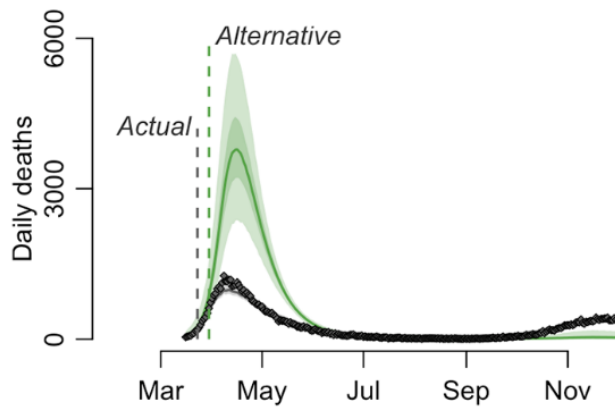

B) Relax lockdown 2 weeks later

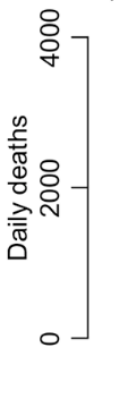

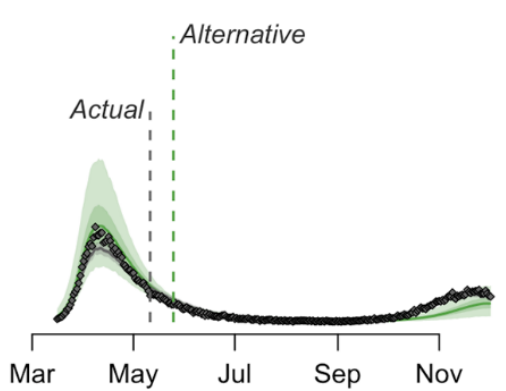

Relax lockdown 2 weeks earlier
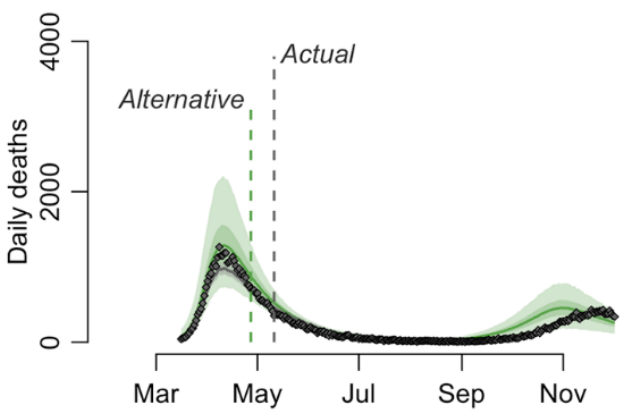

C) $50 \%$ reduced care home visits

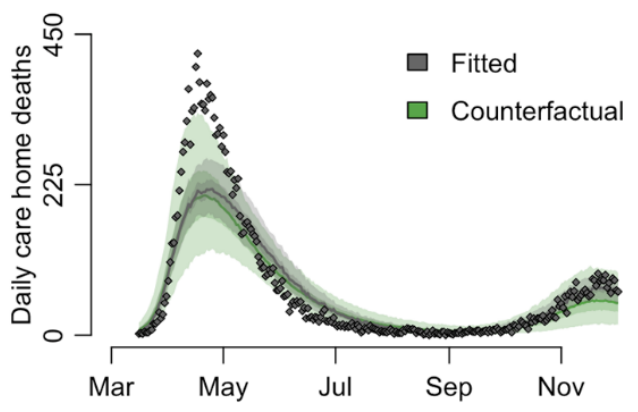

$50 \%$ increased care home visits

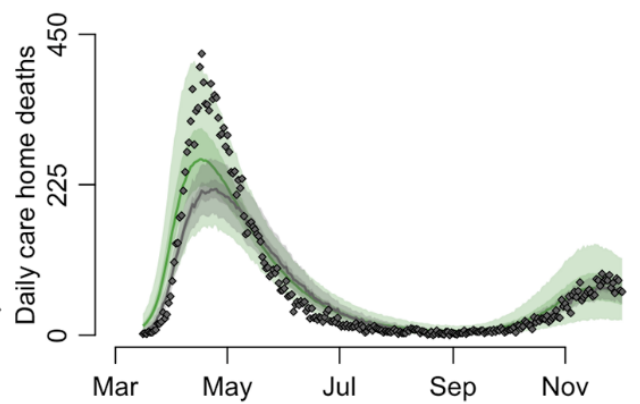

Fig. 6. Counterfactual analysis of the impact on mortality aggregated across NHS England regions. We estimated the impact of (A) initiating lockdown one week earlier / later, (B) Relaxing lockdown two weeks earlier / later, and (C) in response to 50\% more / less restricted care home visits from March to November. (A) and (B) present counterfactual outcomes for daily deaths in England but have different $y$-axis scales to better highlight differences between the observed data and each alternative lockdown scenario. In all panels grey dots depict data (see supplementary materials section 1.1 .2 for details of data sources $(1)(<i>1</ i>)(<i>1</ i>))$. Grey and green solid lines show the posterior median for the fitted and counterfactual model respectively, and shaded bands depict the corresponding 95\% $\mathrm{Crl}$ and interquartile ranges. Vertical dashed lines indicate the timings of the actual and alternative (used in the counterfactual analysis) interventions respectively. Fig. S3 presents a regional breakdown of this figure. 